\title{
Dyons in affine field theories
}

A. C. Cadavid, and R. J. Finkelstein

Citation: Journal of Mathematical Physics 30, 2674 (1989); doi: 10.1063/1.528499

View online: http://dx.doi.org/10.1063/1.528499

View Table of Contents: http://aip.scitation.org/toc/jmp/30/11

Published by the American Institute of Physics

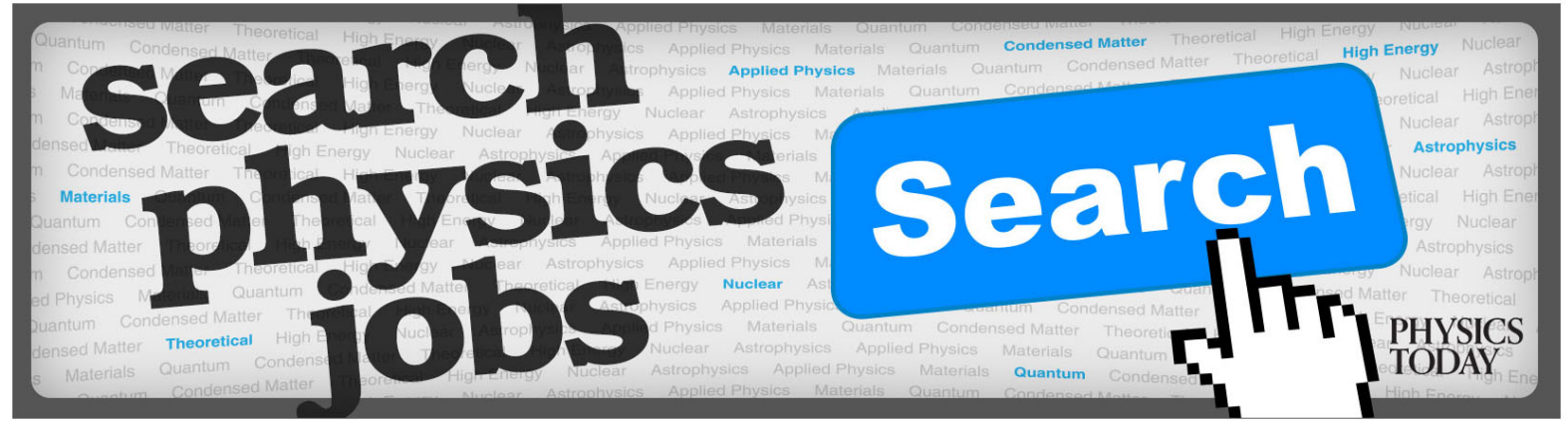




\title{
Dyons in affine field theories
}

\author{
A. C. Cadavid and R. J. Finkelstein \\ Department of Physics, University of California, Los Angeles, California 90024-1547
}

(Received 28 February 1989; accepted for publication 17 May 1989)

By postulating that the gauge vector field and the Higgs field both lie in an affine algebra rather than a Lie algebra, an affine field theory with an expanded soliton sector is obtained. An infinite family of exact, special solutions of the new equations corresponding to the original 't Hooft-Polyakov solitonic solutions, is now found. A perturbation method for an arbitrary solution of the new equation is also proposed.

\section{INTRODUCTION}

Since the work of 't Hooft and Polyakov, it has been known that non-Abelian field theories may have a soliton sector. In particular, magnetic monopoles ${ }^{1,2}$ as well as dyons $^{3}$ may arise in a spontaneously broken $\mathrm{SU}(2)$ gauge theory. Although there is no present evidence for these particles one can speculate that they are simply too heavy to have been observed or that they are bound preons. ${ }^{4}$ If one is interested in gravitationally coupled theories, however, and therefore in extremely high energies, perhaps one should think more about these objects.

The theoretically known Yang-Mills solitons are nodeless, but in principle there could be noding solutions like the excited states of an atom or nucleus. In fact, one should in general expect higher modes of excitation in any spatially extended structure. ${ }^{5}$ It may be, however, that the ensemble of higher modes is realized physically only if one passes from the finite Lie algebra to an infinite algebra. Moreover, such a formal extension is natural since there exists an infinite-dimensional Lie algebra corresponding to every semisimple Lie algebra. This formal extension is also suggested by the idea of regarding the infinite algebra and the corresponding internal space as a surrogate for particle extension in spacetime.

If one does pass from Yang-Mills theories to their affine extensions, ${ }^{6}$ one finds that the soliton sector is indeed correspondingly expanded. In this paper these new solitons will be studied. In taking this step our basic assumption is that the Higgs field, as well as the vector field, lies in a loop algebra rather than in a Lie or in a Kac-Moody algebra. In an earlier note $^{7}$ we discussed the Higgs splitting of a Kac-Moody field and found that the mass of the vector particle was linear in the loop index $n$. That result provided a simple interpretation of $n$ as a label of the heavy vectors, but our assumptions here will be slightly different: for simplicity we limit the discussion to the loop rather than the full Kac-Moody algebra.

\section{FORMULATION} algebra

We assume that the vector and Higgs field lie in the loop

$$
\begin{aligned}
& W_{\mu}=\sum\left(W_{\mu}\right)_{n}{ }^{a} T_{n}{ }^{a}, \\
& \Phi=\sum(\Phi)_{n}{ }^{a} T_{n}{ }^{a},
\end{aligned}
$$

where

$$
\left(T_{n}{ }^{a}, T_{m}{ }^{b}\right)=f^{a b c} T_{n+m}^{c}
$$

and the structure constants are real. Then

$$
\left(T_{n}{ }^{a}\right)^{+}=-T_{-n}^{a} .
$$

We also take both fields to be anti-Hermitian:

$$
\begin{aligned}
& W_{\mu}^{+}=-W_{\mu}, \\
& \Phi^{+}=-\Phi .
\end{aligned}
$$

Then

$$
\begin{aligned}
& \left(W_{\mu}\right)_{n}{ }^{a}=\left(W_{\mu}\right)_{-n}^{a}, \\
& \Phi_{n}{ }^{a}=\Phi_{-n}^{a},
\end{aligned}
$$

if we take the component fields to be real. We consider the action

$$
\begin{aligned}
S= & \int d^{4} x L, \\
L= & \left\langle-\frac{1}{4} G_{\mu \lambda}\left(G^{\mu \lambda}\right)^{+}+\frac{1}{2}\left(D_{\mu} \Phi\right)\left(D^{\mu} \Phi\right)^{+}\right\rangle \\
& +\lambda V\left(\left\langle\Phi^{+} \Phi\right\rangle\right),
\end{aligned}
$$

where \langle\rangle is the invariant scalar product for the algebra, $G_{\mu \lambda}$ is the usual field strength, and $D_{\mu} \Phi$ is a covariant derivative:

$$
\begin{aligned}
& G_{\mu \lambda}=\left(\nabla_{\mu}, \nabla_{\lambda}\right), \\
& D_{\mu} \Phi=\left(\nabla_{\mu}, \Phi\right), \\
& \nabla_{\mu}=\partial_{\mu}+W_{\mu} .
\end{aligned}
$$

Here $W_{\mu}$ and $\Phi$ are the loop vector and scalar fields.

The equations of motion are formally the same as for a Lie algebra since \langle\rangle shares with the trace the properties

$$
\begin{aligned}
& \langle A B\rangle=\langle B A\rangle, \\
& \langle(A, B) C\rangle=\langle(B, C) A\rangle .
\end{aligned}
$$

Also

$$
\left\langle T_{n}{ }^{a} T_{m}{ }^{b}\right\rangle=-\delta^{a b} \delta(n+m) .
$$

The equations of motion are

$$
\begin{aligned}
& \left(\nabla_{\lambda}, G^{\mu \lambda}\right)=\left(\Phi,\left(\nabla^{\mu}, \Phi\right)\right), \\
& \left(\nabla^{\mu},\left(\nabla_{\mu}, \Phi\right)\right)=\lambda \frac{\partial V}{\partial \Phi} .
\end{aligned}
$$

In addition there is the Bianchi identity

$$
\left(\nabla_{\mu}, \widetilde{G}^{\mu \lambda}\right)=0,
$$

where $\widetilde{G}^{\mu \lambda}$ is the dual field. The symmetric energy momentum tensor is 


$$
T^{\mu \lambda}=\left\langle G^{\mu \sigma}\left(G_{\sigma}{ }^{\lambda}\right)^{+}+\left(D^{\mu} \Phi\right)\left(D^{\lambda} \Phi\right)^{+}+g^{\mu \lambda} L\right\rangle
$$

and the energy density is

$$
\begin{aligned}
T^{\infty}= & \frac{1}{2}\left\langle\mathscr{C}^{i}\left(\mathscr{C}^{i}\right)^{+}+\mathscr{B}^{i}\left(\mathscr{B}^{i}\right)^{+}\left(D^{0} \Phi\right)\left(D^{0} \Phi\right)^{+}\right. \\
& \left.+\left(D^{i} \Phi\right)\left(D^{i} \Phi\right)^{+}\right\rangle+\lambda V\left(\left\langle\Phi \Phi^{+}\right\rangle\right),
\end{aligned}
$$

where

$$
\begin{aligned}
& G^{0 i}=\mathscr{C}^{i}, \\
& G^{i j}=\epsilon^{i j k} \mathscr{B}^{k}, \\
& \lambda V>0 .
\end{aligned}
$$

A field configuration for which $T^{00}=0$ everywhere will be called a vacuum configuration. Then by (2.21) a vacuum configuration implies

$$
\begin{aligned}
& G^{\mu \lambda}=0, \\
& D^{\mu} \Phi=0, \\
& V=0 .
\end{aligned}
$$

A region of space-time will be described as a Higgs vacuum if (2.26) and (2.27) but not necessarily (2.25) are satisfied. ${ }^{8}$ The condition of finite energy for the entire soliton field will enforce the Higgs vacuum asymptotically at large distances.

One may ask for classical solitons that solve Eqs. (2.17)-(2.19) subject to the boundary conditions (2.26) and (2.27). Solutions are known where $G^{\mu \lambda}$ and $\Phi$ are both isotriplets lying in $\mathrm{SO}(3)$. For definiteness we limit our work to the loop generalization of $\mathrm{SO}(3)$. Therefore we shall investigate the generalization of the SO(3) solitons to the corresponding loop algebra.

\section{THE HIGGS VACUUM AND THE ELECTROMAGNETIC FIELD:}

In the Higgs vacuum the $\mathrm{SO}(3)$ symmetry is broken down to $S O(2)$ or equivalently to $U(1)$. Then the photon, remaining massless, is separated from the massive vectors which acquire mass $a e \hbar$, where $a=|\phi|$ in the Higgs vacuum and $e$ is the gauge coupling constant. The Higgs particle acquires the mass $a(2 \lambda)^{1 / 2} \hbar$. In the same region of space-time where the Higgs field points in a fixed direction $\phi$, the electromagnetic potential may be identified with $\phi \mathrm{W}^{\mu} / a$ and the electric charge operator with $e \phi \mathrm{T} \hbar / a$, where $T^{a}(a=1,2,3)$ are the $\mathrm{SO}(3)$ generators and $\phi \mathrm{T} / a$ is the equivalent $\mathrm{U}(1)$ generator. In this region the electromagnetic field is well defined. However, the expression for it is not unique since the electromagnetic field is not well defined in the interior of the soliton, although all such expressions must agree in the Higgs vacuum. is 1

The electromagnetic field tensor proposed by 't Hooft

$$
F_{\mu \lambda}=\hat{\phi}^{a}\left(G_{\mu \lambda}^{a}-e^{-1} \epsilon^{a b c} D_{\mu} \hat{\phi}_{b} D_{\lambda} \hat{\phi}_{c}\right),
$$

where

$$
\hat{\phi}^{a}=\phi^{a} /|\phi| .
$$

For $\mathrm{SO}(3)$ this tensor may be rewritten as

$$
F_{\mu \lambda}=M_{\mu \lambda}+H_{\mu \lambda},
$$

where

$$
\begin{aligned}
& M_{\mu \lambda}=\partial_{\mu} A_{\lambda}-\partial_{\lambda} A_{\mu}, \\
& A_{\lambda}=\hat{\phi}^{a} W_{\lambda}{ }^{a},
\end{aligned}
$$

and

$$
H_{\mu \lambda}=e^{-1} \epsilon^{a b c} \hat{\phi}^{a} \partial_{\mu} \hat{\phi}^{b} \partial_{\lambda} \hat{\phi}^{c} .
$$

The magnetic current is

$$
k_{\mu}=\partial^{\lambda} \widetilde{F}_{\mu \lambda},
$$

where $\widetilde{F}_{\mu \lambda}$ is the dual field. Then

$$
\begin{aligned}
k_{\mu}= & \frac{1}{2} \epsilon_{\mu \nu \rho \sigma} \partial^{\nu} M^{\rho \sigma} \\
& -(1 / 2 e) \epsilon_{\mu \nu \rho \sigma} \epsilon^{a b c} \partial_{\nu} \hat{\phi}^{a} \partial_{\rho} \hat{\phi}^{b} \partial_{\sigma} \hat{\phi}^{c} .
\end{aligned}
$$

The magnetic current may arise from the first term if $A_{\lambda}$ is singular on Dirac strings or entirely from the second term if $A_{\lambda}$ has no string singularities. These equivalent descriptions of magnetic charge are connected by a singular gauge transformation. If there are no string singularities, the magnetic charge is

$$
\begin{aligned}
g & =\frac{1}{4 \pi} \int k_{0} d^{3} x \\
& =\frac{1}{8 \pi e} \int \epsilon_{i j k} \epsilon_{a b c} \partial_{i} \hat{\phi}^{a} \partial_{j} \hat{\phi}^{b} g_{k} \hat{\phi}^{c} d^{3} x
\end{aligned}
$$

or

$$
e g=\lim _{R \rightarrow \infty} \frac{1}{8 \pi} \int_{S_{R}^{(2)}} \epsilon_{i j k} \epsilon_{a b c} \hat{\phi}^{a} \partial_{j} \hat{\phi}^{b} \partial_{k} \hat{\phi}^{c}\left(d^{2} \sigma\right)_{i} .
$$

The integral in the preceding section is a topological invariant that can assume only integral values. The total magnetic charge is therefore

$$
g=n / e,
$$

where $n$ is the number of nodes in the Higgs function. ${ }^{9}$

Equation (3.1) for the electromagnetic field is gauge invariant and reduces by (2.26) to the required form in the Higgs vacuum. As already remarked, however, there is no unique definition of $F_{\mu \lambda}$ outside the Higgs vacuum. Another proposal that agrees with (3.1) in the Higgs vacuum is ${ }^{10}$

$$
F_{\mu \lambda}=\boldsymbol{\phi} \cdot \mathbf{G}_{\mu \lambda} / a
$$

or

$$
F_{\mu \lambda}=\operatorname{Tr}\left(\Phi G_{\mu \lambda} / a\right) .
$$

We shall generalize (3.13b) for the loop algebra to

$$
F_{\mu \lambda}=\left\langle\Phi^{+} G_{\mu \lambda}\right\rangle / a,
$$

where

$$
a^{2}=\lim _{r \rightarrow \infty}\left\langle\Phi^{+} \Phi\right\rangle
$$

and \langle\rangle is the invariant scalar product for the loop algebra. Here $F_{\mu \lambda}$ is the generalized electromagnetic field, but only $n=0$ is the Maxwell field. The corresponding generalized magnetic current is

$$
k^{\mu}=\partial_{\lambda}\left\langle\Phi^{+} \widetilde{G}^{\mu \lambda}\right\rangle / a
$$

or 


$$
=\left\langle D_{\lambda} \Phi^{+} \widetilde{G}^{\mu \lambda}\right\rangle / a,
$$

by the Bianchi identities (2.19).

The conservation of $k^{\mu}$ follows from its definition (3.15a) as the divergence of an antisymmetric tensor.

The generalized electric and magnetic charges following from (3.14) are

$$
\begin{aligned}
Q & =\lim _{r \rightarrow \infty} \frac{r^{2}}{a} \int d \Omega\left\langle\Phi^{+} \mathscr{C}_{r}\right\rangle \\
& =\frac{1}{a} \int d^{3} x\left\langle D_{i} \Phi^{+} \mathscr{C}_{i}\right\rangle, \\
M & =\lim _{r \rightarrow \infty} \frac{r^{2}}{a} \int d \Omega\left\langle\Phi^{+} \mathscr{B}_{r}\right\rangle \\
& =\frac{1}{a} \int d^{3} x\left\langle D_{i} \Phi^{+} \mathscr{B}_{i}\right\rangle,
\end{aligned}
$$

where $\mathscr{E}$ and $\mathscr{B}$ are given by (2.22) and (2.23), respectively.

The actual electric and magnetic charges are, of course, given by the $n=0$ components.

\section{DYONS ${ }^{3.11}$} tions

We consider those special solutions obeying the condi-

$$
\begin{aligned}
& \left(W^{0}, \Phi\right)=0, \\
& \partial_{0} W^{\mu}=\partial_{0} \Phi=0 .
\end{aligned}
$$

Then

$$
D_{0} W^{0}=D_{0} \Phi=0 .
$$

With these assumptions it is shown in the Appendix that Eqs. (2.17) $-(2.19)$ are equivalent to the set

$$
\begin{aligned}
& \mathscr{C}_{i}=\sin \alpha D_{i} \Phi, \\
& \mathscr{B}_{i}=\cos \alpha D_{i} \Phi,
\end{aligned}
$$

in the limit $\lambda \rightarrow 0^{+}$. This reduction holds for any algebra including loop algebras.

The mass of the dyon is

$$
m=\int T_{00} d^{3} x .
$$

By (2.21) and (4.3),

$$
m=\frac{1}{2} \int\left\langle\mathscr{C}_{i} \mathscr{C}_{i}{ }^{+}+\mathscr{B}_{i} \mathscr{B}_{i}{ }^{+}+D_{i} \Phi D_{i} \Phi^{+}\right\rangle d^{3} x
$$

By (4.4) and (4.5),

$$
\begin{aligned}
m & =\int\left\langle D_{i} \Phi D_{i} \Phi^{+}\right\rangle d^{3} x \\
& =\int\left\langle\mathscr{C}_{i} \mathscr{C}_{i}{ }^{+}+\mathscr{B}_{i} \mathscr{B}_{i}{ }^{+}\right\rangle d^{3} x .
\end{aligned}
$$

From (3.16), (3.17) and (4.4), (4.5), one finds

$$
Q \sin \alpha=\frac{1}{a} \int\left\langle\mathscr{E}_{i} \mathscr{C}_{i}{ }^{+}\right\rangle d^{3} x,
$$

$$
M \cos \alpha=\frac{1}{a} \int\left\langle\mathscr{B}_{i} \mathscr{B}_{i}{ }^{+}\right\rangle d^{3} x,
$$

and

$$
m=a(Q \sin \alpha+M \cos \alpha) .
$$

Also

$$
\begin{aligned}
& a Q=\sin \alpha \int\left(D_{i} \Phi\right)\left(D_{i} \Phi^{+}\right) d^{3} x \\
& a M=\cos \alpha \int\left(D_{i} \Phi\right)\left(D_{i} \Phi^{+}\right) d^{3} x, \\
& Q / M=\tan \alpha .
\end{aligned}
$$

By (4.11) and (4.14),

$$
m=a\left(Q^{2}+M^{2}\right)^{1 / 2} .
$$

This relation between mass and generalized electric and magnetic charge holds for an exact solution of the affine field equations, and in relation to the finite algebra it is known as the Bogomolny bound. ${ }^{8,12}$

The solution found by Prasad and Sommerfield, a special solution of the general loop equations, is the following $^{8,11}$ :

$$
\begin{aligned}
& e W_{a}^{i}=[K(r)-1] \epsilon_{a i j} \hat{r}^{j} / r, \\
& e \phi_{a}=(H(r) / r) \hat{r}^{a}, \\
& W_{a}{ }^{0}=(J(r) / r) \hat{r}^{a},
\end{aligned}
$$

where

$$
\begin{aligned}
& K(r)=\lambda r / \sinh (\lambda r), \\
& H(r) \cos \alpha=\lambda r \operatorname{coth}(\lambda r)-1, \\
& J(r)=H(r) \sin \alpha,
\end{aligned}
$$

and

$$
\lambda=a e \cos \alpha .
$$

Then

$$
\lim _{r \rightarrow \infty} \phi(r)=a \hat{r} .
$$

The differential equations for the functions $J, H$, and $K$ are scale invariant. The scale of the soliton is fixed by the value of $a$, the vacuum expectation value of the Higgs field.

\section{GENERALIZATION TO LOOP ALGEBRA}

As already noted, Eqs. (4.4) and (4.5) hold for the loop algebra. We again assume (4.1) and (4.2) as well. Then

$$
\mathscr{C}_{k}=G_{0 k}=-D_{k} W_{0}
$$

By (4.4)

$$
D_{k} W_{0}=-\sin \alpha D_{k} \Phi,
$$

so that we may take $W_{0}$ proportional to the Higgs potential as shown in (4.21). Then it is only necessary to solve (4.5).

We make the simplest generalization of the Yang-Wu ansatz $^{13}$ :

$$
\begin{aligned}
& e W_{k}^{a n}=g_{n}(r) \epsilon^{a}{ }_{k l} X^{l}, \\
& e W_{0}^{a n}=J_{n}(r) X^{a}, \\
& e \Phi^{a n}=h_{n}(r) X^{a} .
\end{aligned}
$$

Then 


$$
\mathscr{B}_{k}{ }^{a n}=\delta_{k}{ }^{a} \mathscr{B}_{1}{ }^{n}+X^{a} X_{k} \mathscr{B}_{2}{ }^{n},
$$

where

$$
\begin{aligned}
& e \mathscr{B}_{1}{ }^{n}=-\left(r \frac{d}{d r}+2\right) g_{n}(r), \\
& e \mathscr{B}_{2}{ }^{n}=\frac{1}{r} \frac{d g_{n}}{d r}+\sum_{p} g_{n-p} g_{p},
\end{aligned}
$$

while

$$
\begin{aligned}
& \left(\nabla_{k}, \Phi^{a n}\right)=\delta_{k}{ }^{a} \mathbf{I}^{n}+X^{a} X_{k} \mathbf{I I}^{n}, \\
& e \mathbf{I}^{n}=h_{n}-r^{2} \sum_{p} g_{n-p} h_{p}, \\
& e \mathrm{II}^{n}=\frac{1}{r} \frac{d h_{n}}{d r}+\sum_{p} g_{n-p} h_{p} .
\end{aligned}
$$

Then (4.5) becomes

$$
\begin{aligned}
& r g_{n}{ }^{\prime}+2 g_{n}=\cos \alpha\left[r^{2} \sum_{p} g_{n-p} h_{p}-h_{n}\right] \\
& \frac{1}{r} g_{n}{ }^{\prime}+\sum_{p} g_{n-p} g_{p}=\cos \alpha\left[\frac{1}{r} h_{n}{ }^{\prime}+\sum_{p} g_{n-p} h_{p}\right]
\end{aligned}
$$

In order to describe a soliton solution, these equations must be satisfied subject to the boundary conditions that all the components of $\Phi$ and $W_{\mu}$ be regular at $r=0$ and that they approach the Higgs vacuum at $r=\infty$. We shall require finiteness and a flat tangent at the origin as well as

$$
\begin{aligned}
& \lim _{r \rightarrow \infty} D_{\mu} \Phi=0, \\
& \lim _{r \rightarrow \infty} \Phi_{m}=a_{m} \hat{r} .
\end{aligned}
$$

Then

$$
\lim _{r \rightarrow \infty}\left\langle\Phi^{+} \Phi\right\rangle=\sum a_{m}^{2}=a^{2} .
$$

\section{SPECIAL SOLUTIONS}

Let us denote the $n$th soliton by the set $(\mathbf{g}, \mathbf{h})$, where $g_{m}=h_{m}=0$ if $|m|>n$ and where

$$
\begin{aligned}
& \mathbf{g}=\left(g_{-n} \cdots g_{0} \cdots g_{n}\right), \\
& \mathbf{h}=\left(h_{-n} \cdots h_{0} \cdots h_{n}\right) .
\end{aligned}
$$

By (2.7) and (2.8), $g_{m}=g_{-m}$ and $h_{m}=h_{-m}$. According to (5.8) and (5.9) the components $\left(g_{0}, h_{0}\right)$ satisfy

$$
\begin{aligned}
& r g_{0}{ }^{\prime}=-2 g_{0}-\bar{h}_{0}+r^{2}\left(g_{0} \bar{h}_{0}+2 \sum_{1}^{n} g_{m} \bar{h}_{m}\right), \\
& r \bar{h}_{0}{ }^{\prime}=-2 g_{0}-\bar{h}_{0}+r^{2}\left(g_{0}{ }^{2}+2 \sum_{1}^{n} g_{m}{ }^{2}\right) .
\end{aligned}
$$

The remaining components satisfy

$$
\begin{aligned}
& r g_{m}{ }^{\prime}=-2 g_{m}-\bar{h}_{m}+r^{2} \sum_{0}^{m} g_{m-p} \bar{h}_{p}, \\
& r \bar{h}_{m}{ }^{\prime}=-2 g_{m}-\bar{h}_{m}+r^{2} \sum_{0}^{m} g_{m-p} g_{p} .
\end{aligned}
$$

Here

$$
\bar{h}_{m}=\cos \alpha h_{m} .
$$

The complete set of components $\left(g_{0} \cdots g_{n} ; h_{0} \cdots h_{n}\right)$ is codetermined by the nonlinear equations $(6.3)-(6.6)$. Notice that $\left(h_{m}, g_{m}\right)$ cannot be renormalized independently. They can be rescaled together, however, if lengths are also rescaled.

The Prasad-Sommerfield solution is recognized in this class of solutions as

$$
\begin{aligned}
& \bar{h}_{m}=g_{m}=0, \quad m \neq 0, \\
& \bar{h}_{0}(r)=\left(1 / r^{2}\right)\left[1-\lambda_{0} \operatorname{coth}\left(\lambda_{0} r\right)\right], \\
& g_{0}(r)=\left(1 / r^{2}\right)\left[1-\lambda_{0} r / \sinh \left(\lambda_{0} r\right)\right],
\end{aligned}
$$

where

$$
\lambda_{0}=a e \cos \alpha .
$$

This solution will be described as a "singlet."

Since all components are coupled nonlinearly, there is no obvious method to investigate the general solution of these equations. One may, however, obtain special solutions in the following way. With $r$ corresponding to the time, the general solution will trace out an orbit in $(\mathbf{h}, \mathbf{g})$ space. We may find special solutions in this space by confining the motion to a subspace of the full space. In particular, we may ask for solutions lying in the following subspace:

$$
\begin{aligned}
& \sum_{1}^{n} g_{m} h_{m}=K g_{0} h_{0}, \\
& \sum_{1}^{n} g_{m}{ }^{2}=K g_{0}{ }^{2} .
\end{aligned}
$$

Then (6.3) and (6.4) become

$$
\begin{aligned}
& r g_{0}{ }^{\prime}=-2 g_{0}-\bar{h}_{0}+(1+2 K) r^{2} g_{0} \bar{h}_{0}, \\
& r \bar{h}_{0}{ }^{\prime}=-2 g_{0}-\bar{h}_{0}+(1+2 K) r^{2} g_{0}{ }^{2} .
\end{aligned}
$$

Now rescale the length by

$$
x^{2}=(1+2 K) r^{2} \text {. }
$$

Then

$$
\begin{aligned}
& x \frac{d g_{0}}{d x}=-2 g_{0}-\bar{h}_{0}+x^{2} g_{0} \bar{h}_{0} \\
& x \frac{d \bar{h}_{0}}{d x}=-2 g_{0}-\bar{h}_{0}+x^{2} g_{0}{ }^{2}
\end{aligned}
$$

These rescaled equations have the explicit solutions for $\left(g_{0}, h_{0}\right)$ given by $(6.9)$ and $(6.10)$ in which $r$ is replaced by $(1+2 K)^{1 / 2} r$. The rescaling, of course, depends on the other components $\left(g_{1} \cdots g_{n}\right)$.

Since they are not integrals of the complete set of equations, (6.12) and (6.13) are nonlinear constraints on the set (6.5) and (6.6). These constraints may be satisfied for the special solutions

$$
\begin{aligned}
& \mathbf{g}_{n}=\left(g_{-n} \circ \cdots \circ g_{0} \circ \cdots \circ g_{n}\right), \\
& g_{m}=0, \quad|m|=1, \ldots, n-1, \\
& \mathbf{h}_{n}=\left(h_{-n} \circ \cdots \circ g_{0} \circ \cdots \circ h_{n}\right), \\
& h_{m}=0, \quad|m|=1, \ldots, n-1 .
\end{aligned}
$$

These special solutions will be termed "triplets." Then (6.12) and (6.13) imply 


$$
\begin{aligned}
& g_{n}=k g_{0}, \\
& \bar{h}_{n}=k \bar{h}_{0},
\end{aligned}
$$

where

$$
k= \pm \sqrt{K}
$$

and

$$
\begin{aligned}
& \sum_{0}^{n} g_{n-p} \bar{h}_{p}=2 k g_{0} \bar{h}_{0}, \\
& \sum_{0}^{n} g_{n-p} g_{p}=2 k g_{0}{ }^{2} .
\end{aligned}
$$

Equations (6.5) and (6.6) for the components $\left(g_{n}, h_{n}\right)$ become

$$
\begin{aligned}
& r g_{0}{ }^{\prime}=-2 g_{0}-\bar{h}_{0}+2 r^{2} g_{0} \bar{h}_{0}, \\
& r \bar{h}_{0}{ }^{\prime}=-2 g_{0}-\bar{h}_{0}+2 r^{2} g_{0}{ }^{2} .
\end{aligned}
$$

These are identical with (6.14) and (6.15) for $\left(g_{0}, \bar{h}_{0}\right)$ with

$$
\begin{aligned}
& 1+2 K=2, \\
& k= \pm \frac{1}{2} \sqrt{2} .
\end{aligned}
$$

Therefore $\left(g_{0}, \bar{h}_{0}\right)$ and $\left(g_{n}, \bar{h}_{n}\right)$ are given by the explicit solutions (6.9) and (6.10) in which $r$ is replaced by $\sqrt{2} r$.

By (6.20) and (6.26), however, $g_{n}$ and $h_{n}$ are normalized differently from $g_{0}$ and $h_{0}$ :

$$
\begin{aligned}
& g_{n}(x)=\frac{1}{2} \sqrt{2} g_{0}(x), \\
& h_{n}(x)=\frac{1}{2} \sqrt{2} h_{0}(x) .
\end{aligned}
$$

We have now found an infinite set of exact solutions. Let us denote their $h$ components by $h_{m}{ }^{(n)}$. The simplest of these is the singlet for which

$$
h_{m}{ }^{(0)}=\left(r^{2} \cos \alpha\right)^{-1}\left(1-\lambda_{0} r \operatorname{coth}\left(\lambda_{0} r\right)\right) \delta_{m 0} .
$$

This is the previously obtained solution given by $(6.8)-$ (6.11).

The new set contains the triplets for which

$$
h_{m}{ }^{(n)}=\delta_{m 0} h_{0}{ }^{(0)}(x)+\frac{1}{2} \sqrt{2} \delta_{n|m|} h_{0}^{(0)}(x), \quad n>0,
$$

where

$$
x=\sqrt{2} r \text {. }
$$

Let us label the boundary values by $a_{m}{ }^{(n)}$. Then, by (5.11),

$$
\lim _{r \rightarrow \infty} r\left|h_{m}{ }^{(n)}(r)\right|=e a_{m}{ }^{(n)},
$$

and, by (6.30), one finds

$$
\begin{aligned}
& a_{0}{ }^{(n)}=\frac{1}{2} \sqrt{2} a_{0}{ }^{(0)}, \\
& a_{n}{ }^{(n)}=a_{-n}{ }^{(n)}=\frac{1}{2} a_{0}{ }^{(0)} .
\end{aligned}
$$

We are also interested in the sums

$$
\begin{aligned}
\left(a^{(n)}\right)^{2} & \equiv \lim _{r \rightarrow \infty}\left\langle\Phi^{(n)}\left(\Phi^{(n)}\right)^{+}\right\rangle \\
& =\sum_{-n}^{n}\left(a_{m}{ }^{(n)}\right)^{2} .
\end{aligned}
$$

For the triplets

$$
\left(a^{(n)}\right)^{2}=\left(a_{0}^{(n)}\right)^{2}+2\left(a_{n}{ }^{(n)}\right)^{2} .
$$

Then by (6.33) and (6.34),

$$
a^{(n)}= \pm a^{(0)}
$$

\section{ELECTRIC AND MAGNETIC CHARGES AND MASS}

The formally generalized electric charge may be calculated by (3.17):

$$
\begin{aligned}
a^{(n)} Q^{(n)} & =\lim _{r \rightarrow \infty} r^{2} \int d \Omega\left\langle\Phi^{+} \mathscr{C}_{r}\right\rangle \\
& =4 \pi \lim _{r \rightarrow \infty} r^{2}\left\langle\Phi^{+} \mathscr{C}_{r}\right\rangle .
\end{aligned}
$$

The scalar product is

$$
\begin{aligned}
\left\langle\Phi^{+} \mathscr{C}_{r}\right\rangle & =\sin \alpha\left\langle\Phi^{+} D_{r} \Phi\right\rangle \\
& =\sin \alpha\left\langle\Phi^{+} \partial_{r} \Phi+\Phi^{+}\left(W_{r}, \Phi\right)\right\rangle .
\end{aligned}
$$

The commutator term depends on

$$
\left\langle\Phi^{+}\left(W_{r}, \Phi\right)\right\rangle=-\left\langle W_{r}(\Phi, \Phi)\right\rangle=0 .
$$

Then

$$
\begin{aligned}
\left\langle\Phi^{+} \mathscr{C}_{r}\right\rangle & =\sin \alpha\left\langle\Phi^{+} \partial_{r} \Phi\right\rangle \\
& =\frac{1}{2} \sin \alpha \sum_{a n} \frac{d}{d r}\left(\Phi_{n}{ }^{a}\right)^{2} .
\end{aligned}
$$

The total charge of the $n$th solution is

$$
Q^{(n)}=\sum_{-n}^{n} Q_{m}^{(n)},
$$

where

$$
a^{(n)} Q_{m}{ }^{(n)}=\frac{2 \pi}{e^{2}} \sin \alpha \lim _{r \rightarrow \infty} r^{2} \frac{d}{d r} r^{2}\left[h_{m}{ }^{(n)}(r)\right]^{2} .
$$

Then for the singlet

$$
a^{(\grave{(})} Q^{(0)}=\frac{2 \pi}{e^{2}} \sin \alpha \lim _{r \rightarrow \infty} r^{2} \frac{d}{d r} r^{2}\left[h_{0}^{(0)}(r)\right]^{2},
$$

where $h_{0}{ }^{(0)}(r)$ is given by $(6.29)$ and

$$
\lambda_{0}=a^{(0)} e \cos \alpha \text {. }
$$

Taking the limit one finds the known result

$$
Q^{(0)}=(4 \pi / e) \tan \alpha .
$$

For the triplets, one has

$$
Q^{(n)}=Q_{0}^{(n)}+2 Q_{n}^{(n)},
$$

where

$$
a^{(n)} Q_{0}{ }^{(n)}=\frac{2 \pi}{e^{2}} \sin \alpha \lim _{r \rightarrow \infty} r^{2} \frac{d}{d r} r^{2}\left(h_{0}{ }^{(n)}(r)\right)^{2} .
$$

By (6.30),

$$
\begin{aligned}
a^{n} Q_{0}^{(n)} & =\frac{2 \pi}{e^{2}} \sin \alpha\left(\frac{1}{\sqrt{2}}\right)^{3} \lim _{x \rightarrow \infty} x^{2} \frac{d}{d x} x^{2}\left(h_{0}^{(0)}(x)\right)^{2} \\
& =(1 / \sqrt{2})^{3} a^{(0)} Q^{(0)}
\end{aligned}
$$

or

$$
Q_{0}^{(n)}=\frac{1}{4} \sqrt{2} Q^{(0)},
$$


by (6.30) and (6.37). The additional contributions to the total triplet charge are $Q_{n}{ }^{(n)}$ and $Q_{-n}{ }^{(n)}$. For these we have

$$
\begin{aligned}
a^{(n)} Q_{ \pm n}{ }^{(n)}= & \frac{2 \pi}{e^{2}} \sin \alpha \lim _{r \rightarrow \infty} r^{2} \frac{d}{d r} r^{2}\left(h_{n}{ }^{(n)}(r)\right)^{2} \\
= & \left(\frac{1}{\sqrt{2}}\right)^{3} \frac{1}{2} \frac{2 \pi}{e^{2}} \sin \alpha \\
& \times \lim _{x \rightarrow \infty} x^{2} \frac{d}{d x} x^{2}\left(h_{0}{ }^{(0)}(x)\right)^{2} \\
& =(\sqrt{2} / 8) a^{(0)} Q^{(0)}, \\
\therefore \quad Q_{ \pm n}{ }^{(n)} & =(\sqrt{2} / 8) Q^{(0)} .
\end{aligned}
$$

Then the total triplet charge is

$$
Q^{(n)}=(\sqrt{2} / 4+2 \sqrt{2} / 8) Q^{(0)}=\frac{1}{2} \sqrt{2} Q^{(0)}
$$

or

$$
Q^{(n)}=(\sqrt{2} / 2)((4 \pi / e) \tan \alpha) .
$$

The corresponding magnetic charge is

$$
M^{(n)}=(\sqrt{2} / 2)(4 \pi / e),
$$

by (4.14).

The true electric and magnetic charges come from the Maxwell field only and for the singlet have the usual values:

$$
\begin{aligned}
& q^{(0)}=Q_{0}{ }^{(0)}=(4 \pi / e) \tan \alpha, \\
& g^{(0)}=4 \pi / e .
\end{aligned}
$$

For the triplets these charges are reduced:

$$
\begin{aligned}
& q^{(n)}=Q_{0}{ }^{(n)}=(\sqrt{2} / 4) q^{(0)}, \\
& g^{(n)}=(\sqrt{2} / 4) g^{(0)} .
\end{aligned}
$$

The mass is determined by the total charge according to (4.14) and (4.15):

$$
m=a Q / \sin \alpha .
$$

Then

$$
\begin{aligned}
& m^{(0)}=a^{(0)} Q^{(0)} / \sin \alpha=a^{(0)} q / \sin \alpha, \\
& m^{(n)}=a^{(n)} Q^{(n)} / \sin \alpha .
\end{aligned}
$$

By (7.15a),

$$
\begin{aligned}
& m^{(n)}=(\sqrt{2} / 2) a^{(0)} Q^{(0)} / \sin \alpha, \\
& \therefore \quad m^{(n)}=(\sqrt{2} / 2) m^{(0)} .
\end{aligned}
$$

The triplet dyons have less charge and also less mass than the singlet. All triplets have the same charges and mass.

\section{GENERAL SOLUTIONS}

The special solutions just discussed are not only exact but are also the simplest. They also span an infinite-dimensional function space, but a general solution of the soliton equations (5.8) and (5.9) does not lie in this space since the soliton equations are nonlinear.

We have also studied a slightly different set of equations obtained by dropping the terms in (6.3) and (6.4) coupling $\left(h_{0}, g_{0}\right)$ to the higher modes $\left(h_{m}, g_{m}\right)$, namely, $\Sigma_{1}^{n} g_{m}{ }^{2}$ and $\Sigma_{1}^{n} g_{m} h_{m}$. In this truncation the remaining equations are to be left unchanged. We propose the so-modified set of equations as the basis of a general perturbation method for investigating arbitrary solutions of the exact equations, provided that the higher amplitudes $\left(h_{m}, g_{m}\right)$ are small compared to the zero set $\left(h_{0}, g_{0}\right)$. The modified equations themselves may be solved exactly by the following procedure.

Let the general solution be

$$
\begin{aligned}
& \vec{g}=\left(g_{-n} g_{-n+1} \cdots g_{0} \cdots g_{n-1} g_{n}\right), \\
& \vec{h}=\left(h_{-n} h_{-n+1} \cdots h_{0} \cdots h_{n-1} h_{n}\right) .
\end{aligned}
$$

where $\left(g_{0}, h_{0}\right)$ is the exact Prasad-Sommerfield solution since $\left(g_{0}, h_{0}\right)$ is now decoupled from the other modes. The remaining components may be shown to satisfy

$$
\begin{aligned}
& g_{m}{ }^{\prime \prime}+P g_{m}{ }^{\prime}+Q_{g} g_{m}=S_{m}{ }^{(g)}, \\
& h_{m}{ }^{\prime \prime}+P h_{m}{ }^{\prime}+Q_{h} h_{m}=S_{m}{ }^{(h)},
\end{aligned}
$$

where

$$
\begin{aligned}
& P=(2 / r) F, \\
& Q_{g}=\left[F^{2}-3 G^{2}-1\right] / r^{2}, \\
& S_{m}^{(g)}=G \sum_{p=1}^{m-1} g_{m-p} g_{p},
\end{aligned}
$$

and

$$
Q_{h}=2\left[F-G^{2}-1\right] / r^{2},
$$

$$
\begin{aligned}
S_{m}{ }^{(h)} & \cos \alpha \\
= & {\left[\left(r \frac{d}{d r}+2 F\right)^{m} \sum_{p=1}^{m-1} g_{m-p} g_{p}+2 G \sum_{p=1}^{m-1} g_{m-p} h_{p}\right] . }
\end{aligned}
$$

These expressions all depend on only the two functions $F$ and $H$, which are linear functionals of $h_{0}$ and $g_{0}$ :

$$
\begin{aligned}
& F=2-r^{2} \cos \alpha h_{0}, \\
& G=r^{2} g_{0}-1 .
\end{aligned}
$$

From $(8.2 \mathrm{~h})$ and $(8.2 \mathrm{~g})$ one sees that $\left(h_{m}, g_{m}\right)$ satisfy linear equations where the coefficients depend on the pair $\left(h_{0}, g_{0}\right)$, which in turn satisfy the nonlinear equations (6.14) and (6.15) with $K=0$. Since these coefficients depend only on $\left(h_{0}, g_{0}\right)$, they are the same for all $m$. The different components are distinguished by only the source terms $\left(S_{m}{ }^{(h)}, S_{m}{ }^{(g)}\right)$, which do not depend on $\left(h_{m}, g_{m}\right)$ but do depend on all the lower components $\left(h_{p}, g_{p}\right)$, where $1 \leqslant p \leqslant m-1$. As a consequence of this structure these coupled equations may be solved by first solving the nonlinear equations for $\left(h_{0}, g_{0}\right)$ to obtain (6.9) and (6.10), and then proceeding along the sequence $\left(h_{1}, g_{1}\right) \cdots\left(h_{m}, g_{m}\right)$ $\cdots\left(h_{n}, g_{n}\right)$ by solving at each step equations that are only linear in the new variables $\left(h_{m}, g_{m}\right)$.

Since there is no source for the $\left(h_{1}, g_{1}\right)$ equation, the required $\left(h_{1}, g_{1}\right)$ functions satisfy homogeneous equations subject to the boundary conditions at the origin and infinity. To proceed to higher states we shall introduce the Green's function, which also satisfies the proper boundary conditions. The general solution of these equations is the sum of the solutions of the homogeneous equation and a special solution determined by the source: 


$$
\begin{array}{rlrl}
g_{m}(r)=\dot{g}_{m}(r)+\int_{0}^{\infty} W(r) G_{g}\left(r, r^{\prime}\right) S_{m}{ }^{(g)}\left(r^{\prime}\right) d r^{\prime}, & h_{1<} & =\frac{1}{\lambda_{0} r}\left[-\operatorname{coth} \lambda_{0} r+\frac{\lambda_{0} \mathrm{r}}{\sinh ^{2} \lambda_{0} r}\right], \\
(8.10 \mathrm{~g}) & h_{1>}=\frac{1}{\lambda_{0} r} \frac{1}{\sinh ^{2} \lambda_{0} r} .
\end{array}
$$$$
h_{m}(r)=\grave{h}_{m}(r)+\int_{0}^{\infty} W(r) G_{h}\left(r, r^{\prime}\right) S_{m}{ }^{(h)}\left(r^{\prime}\right) d r^{\prime} .
$$

Since the differential equations are not Sturm-Liouville, it is necessary to introduce a weight function $W(r)$ independent of $m$ :

$$
W(r)=(r \sinh r)^{2} .
$$

The Green's functions are also independent of $m$ since they depend on only $\left(h_{1}, g_{1}\right)$ as follows:

$$
\begin{aligned}
& G_{g}\left(r, r^{\prime}\right)= \begin{cases}-g_{1<}\left(r^{\prime}\right) g_{1>}(r), & r>r^{\prime}, \\
-g_{1>}\left(r^{\prime}\right) g_{1<}(r), & r<r^{\prime} ;\end{cases} \\
& G_{h}\left(r, r^{\prime}\right)= \begin{cases}\frac{1}{2} h_{1<}\left(r^{\prime}\right) h_{1>}(r), & r>r^{\prime}, \\
\frac{1}{2} h_{1>}\left(r^{\prime}\right) h_{1<}(r), & r<r^{\prime} ;\end{cases}
\end{aligned}
$$

where a subscript $<$ refers to solutions of homogeneous equations satisfying boundary conditions at the origin and a subscript $>$ refers to an independent solution satisfying boundary conditions at infinity.

\section{THE LOWEST STATES OF THE MODIFIED EQUATIONS}

The first excited state is characterized by $(\mathbf{h}, \mathbf{g})$, where $\mathbf{h}=\left(h_{0}, h_{1}\right)$ and $\mathbf{g}=\left(g_{0}, g_{1}\right)$. The components $\left(h_{0}, g_{0}\right)$ have already been given in (6.9) and (6.10). The new components satisfy the homogeneous linear equations

$$
\begin{aligned}
& g_{1}{ }^{\prime \prime}+P g_{1}{ }^{\prime}+Q_{g} g_{1}=0, \\
& h_{1}{ }^{\prime \prime}+P h^{\prime}+Q_{h} h_{1}=0,
\end{aligned}
$$

with solutions of the form

$$
\begin{aligned}
& g_{1}(r)=\exp \left[-\frac{1}{2} \int^{r} P(s) d s\right] U_{g}(r), \\
& h_{1}(r)=\exp \left[-\frac{1}{2} \int^{r} P(s) d s\right] U_{h}(r),
\end{aligned}
$$

where

$$
\begin{aligned}
& U_{g}^{\prime \prime}+\left(Q_{g}-\frac{1}{2} P^{\prime}-\frac{1}{4} P^{\prime \prime}\right) U_{g}=0, \\
& U_{h}^{\prime \prime}+\left(Q_{h}-\frac{1}{2} P^{\prime}-\frac{1}{4} P^{\prime \prime}\right) U_{h}=0 .
\end{aligned}
$$

By (8.3), (8.4), and (8.6), one finds

$$
\begin{aligned}
& P=(2 / r)\left(1+\lambda_{0} r \operatorname{coth} \lambda_{0} r\right), \\
& Q_{g}-\frac{1}{2} P^{\prime}-\frac{1}{4} P^{2}=-2\left(\lambda_{0} / \sinh \lambda_{0} r\right)^{2}, \\
& Q_{h}-\frac{1}{2} P^{\prime}-\frac{1}{4} P^{2}=-\lambda_{0}{ }^{2}-2\left(\lambda_{0} / \sinh \lambda_{0} r\right)^{2} .
\end{aligned}
$$

There are two independent solutions of both the $h$ and $g$ equations. These may be chosen to be

$$
\begin{aligned}
& g_{1<}=\frac{1}{\sinh \lambda_{0} r}\left(\operatorname{coth} \lambda_{0} r-\frac{1}{\lambda_{0} r}\right), \\
& g_{1>}=\frac{1}{\lambda_{0} r} \frac{\operatorname{coth} \lambda_{0} r}{\sinh \lambda_{0} r}
\end{aligned}
$$

and
Here $g_{1<}$ and $h_{1<}$ satisfy the boundary conditions of regularity at the origin; these functions also vanish properly at infinity. On the other hand, $g_{1>}$ and $h_{1>}$ vanish at infinity but blow up at the origin. The solutions $g_{1<}$ and $h_{1<}$ satisfy not only the second-order equations (9.1) but also the firstorder equations (6.5) and (6.6), as required. The functions $g_{1<}$ and $h_{1<}$ therefore describe the approximate solitonic solution we seek.

Since both the first- and second-order equations are linear and homogeneous in $g_{1}$ and $h_{1}$, these equations do not fix the normalization of these functions. However, since the first-order equations (6.5) and (6.6) relate the normalization of $g_{1}$ and $h_{1}$, only one normalization condition needs to be supplied. We take this to be the boundary condition at infinity for $h_{1<}$. At the two limits we have

$$
\begin{aligned}
& \lim _{r \rightarrow 0} g_{1<}\left(\lambda_{0} r\right)=\frac{1}{3} A_{1}, \\
& \lim _{r \rightarrow 0} h_{1<}\left(\lambda_{0} r\right)=\frac{2}{3} A_{1},
\end{aligned}
$$

where $A_{1}$ normalizes $g_{1<}$ and $h_{1<}$ and

$$
\begin{aligned}
& \lim _{r \rightarrow \infty} g_{1<}\left(\lambda_{0} r\right)=A_{1} e^{-\lambda_{0} r}, \\
& \lim _{r \rightarrow \infty}\left|h_{1<}\left(\lambda_{0} r\right)\right|=A_{1} / \lambda_{0} r .
\end{aligned}
$$

But we require, by (5.11),

$$
\lim _{r \rightarrow \infty} r\left|h_{n}(r)\right|=e a_{n}
$$

or

$$
\begin{aligned}
& A_{1}=\lambda_{0} \lambda_{1}, \\
& \lambda_{1}=a_{1} e \cos \alpha .
\end{aligned}
$$

The approximate soliton $\left(h_{0}, g_{0} ; h_{1}, g_{1}\right)$ is then entirely determined by the parameters appearing in the action and the constants $\left\{a_{m}\right\}$, the vacuum expectation values of the extended Higgs field.

The $\left(h_{2}, g_{2}\right)$ solutions of the modified equations are

$$
\begin{aligned}
h_{2}= & \frac{1}{\cos \alpha} \frac{1}{y}\left[\left(A_{2}+\lambda_{1}{ }^{2}\right) \frac{y}{\sinh ^{2} y}\right. \\
& \left.-\lambda_{1}{ }^{2} \frac{y^{2} \operatorname{coth} y}{\sinh ^{2} y}-A_{2} \operatorname{coth} y\right], \\
g_{2}= & \left(A_{2}+\lambda_{1}^{2}\right) \frac{\operatorname{coth} y}{\sinh y}-A_{2} \frac{1}{y \sinh y} \\
& -\frac{\lambda_{1}{ }^{2}}{2}\left[\frac{y}{\sinh ^{3} y}+\frac{y \operatorname{coth}^{2} y}{\sinh y}\right],
\end{aligned}
$$

where $y=\lambda_{0} r$ and $A_{2}$ is the constant that multiplies the solution of the homogeneous equation. The value of $A_{2}$ is fixed by the limit of $h_{2}$ at large distances. By (9.13), $A_{2}=\lambda_{0} \lambda_{2}$, with $\lambda_{2}=a_{2} e \cos \alpha$.

There is no reason for this series of solutions to termi- 
nate. The recursive procedure for generating new solutions is described in Sec. VIII. The explicit form of the Green's function appearing there is obtained by substituting the appropriate $h$ and $g$ solutions from (9.7)-(9.10) into (8.12). The general solution is the sum of solutions of the homogeneous equation and the special solution depending on the source and the Green's function.

At each stage of this recursive procedure one must determine the constant associated with the solution to the homogeneous equation. In the next step we encounter the constant $A_{3}=\lambda_{0} \lambda_{3}$ with $\lambda_{3}=a_{3} e \cos \alpha$.

Although we do not have a general formula for the solutions at any level $n$, only the contribution from the homogeneous solution is important in the calculation of (9.13).

For $n=3$, we have

$$
\begin{aligned}
h_{3}= & \frac{1}{\cos \alpha}\left[\frac{1}{y}\left[\left(A_{3}+2 \lambda_{2} \lambda_{1}\right) \frac{y}{\sinh ^{2} y}-A_{3} \operatorname{coth} y\right]\right. \\
& -\left(\frac{\lambda_{1}{ }^{3}}{\lambda_{0}}+2 \lambda_{1} \lambda_{2}\right) \frac{y \operatorname{coth} y}{\sinh ^{2} y} \\
& \left.+\frac{\lambda_{1}^{3}}{\lambda_{0}}\left[\frac{y^{2} \operatorname{coth}^{2} y}{\sinh ^{2} y}-\frac{1}{3} \frac{y^{2}}{\sinh ^{2} y}\right]\right] \\
g_{3}= & \left(A_{3}+2 \lambda_{2} \lambda_{1}\right) \frac{\operatorname{coth} y}{\sinh y}-A_{3} \frac{1}{y \sinh y} \\
& -\left(\lambda_{2} \lambda_{1}+\frac{1}{2} \frac{\lambda_{1}^{3}}{\lambda_{0}}\right)\left[\frac{2 y}{\sinh ^{3} y}+\frac{y}{\sinh y}\right] \\
& +\frac{\lambda_{1}^{3}}{\lambda_{0}}\left(\frac{y^{2} \operatorname{coth}^{2} y}{\sinh ^{3} y}+\frac{1}{6} \frac{y^{2} \operatorname{coth} y}{\sinh y}\right)
\end{aligned}
$$

For $n=1$ there is a test of this perturbative procedure since we have both the exact solution and the approximate solution as determined from the modified equations. For small $r$ the approximate solution (9.9) becomes

$$
h_{1}^{(1)} \sim\left(1 /\left(\lambda_{0} r\right)^{2}\right)\left[\left(\lambda_{0} r\right) \operatorname{coth}\left(\lambda_{0} r\right)-1\right] .
$$

This expression is of the same form as the exact solution (6.30). At large distances, on the other hand, the exact and approximate solutions do not have the same form; but at large distances one has a linear approximation to the unmodified equations.

In general, if the higher $n$ components are small compared to $\left(h_{0}, g_{0}\right)$, one would expect the preceding procedure based on the modified equations to be useful.

\section{$X$. REMARKS}

In order to investigate the dependence of the soliton sector on the affine index, we have studied a very special model that can be generalized in different ways. In particular, if the total field contained an additional component tranforming according to some irreducible and higher-dimensional representation of $\mathrm{SU}(2)$, this additional component carrying electric charge would interact with the magnetic charge so as to convert internal degrees of freedom into spin degrees of freedom with the result that the total angular momentum would no longer vanish; the total angular momentum would then be either integral or half integral, depending on the transformation properties of the additional field. ${ }^{14,15}$ One would also expect that this additional structure might lift the infinite mass degeneracy of the triplet solitons. Then the members of the infinite family of dyons would differ not only in mass but also in angular momentum and statistics, ${ }^{16}$ and in a way that can be calculated once the model is made definite.

The refinements just described have not yet been investigated. We have shown elsewhere, however, how the theory may be made globally ${ }^{17}$ and also locally ${ }^{18}$ supersymmetric.

The preceding comments refer to the soliton sector of the theory, which one may expect to be excited only at very high energies. At currently accessible energies, on the other hand, the vacuum or "meson" sector provides the appropriate formalism. Here the particle states have spin 1 and 0 . In this sector, where one has the standard procedures, we have previously studied the Higgs splitting and found a linear mass spectrum for the vectors if the Higgs has a component lying in the $L_{0}$ direction of the associated Virasoro algebra. In the present paper our model is different and our object has been to concentrate on the affine solitons rather than to provide a unified consistent model for both sectors. One may, however, plausibly assume that the soliton and vacuum sectors exhibit different representations of the Kac-Moody algebra and, in particular, that the central operator $k$ and the Virasoro operator $L_{0}$ vanish for the soliton sector. A more realistic model would also address this question.

\section{APPENDIX: DERIVATION OF EQS. (4.4) AND (4.5)}

Equations (4.4) and (4.5) may be obtained from the field equations in the following way. If we distinguish space from time coordinates, the set $(2.17)-(2.19)$ becomes

$$
\begin{aligned}
& \left(\nabla_{k}, G^{0 k}\right)=0, \\
& \left(\nabla_{k}, \widetilde{G}^{0 k}\right)=0, \\
& \left(\nabla_{k},\left(\nabla^{k}, \Phi\right)\right)=0, \\
& \left(\nabla_{\lambda}, \widetilde{G}^{k \lambda}\right)=0, \\
& \left(\nabla_{\lambda}, G^{k \lambda}\right)=\left(\Phi^{+},\left(\nabla^{k}, \Phi\right)\right) .
\end{aligned}
$$

Let us try

$$
\begin{aligned}
& G_{k 0}=a\left(\nabla_{k}, \Phi\right), \\
& \widetilde{G}_{k 0}=b\left(\nabla_{k}, \Phi\right) .
\end{aligned}
$$

By (A6) and (A7), Eqs. (A1)-(A3) become equivalent. Then it is enough to check (A2). But (A2) and (A4) together comprise the Bianchi identity. Since both may be checked in the same way, we write out (A4) only.

One equation of the set (A4) is

$$
\left(\nabla_{0}, \widetilde{G}^{10}\right)+\left(\nabla_{2} \widetilde{G}^{12}\right)+\left(\nabla_{3} \widetilde{G}^{13}\right)=0
$$

or

$$
\left(\nabla_{0}, \widetilde{G}_{01}\right)+\left(\nabla_{2}, G_{03}\right)+\left(\nabla_{3}, G_{20}\right)=0 .
$$

By (A6) and (A7),

$$
-b\left(\nabla_{0},\left(\nabla_{1}, \Phi\right)\right)-a\left(\nabla_{2},\left(\nabla_{3}, \Phi\right)\right)+a\left(\nabla_{3},\left(\nabla_{2}, \Phi\right)\right)=0 \text {. }
$$

By the Jacobi identity,

$$
-b\left(\nabla_{0},\left(\nabla_{1}, \Phi\right)\right)+a\left(\Phi,\left(\nabla_{2}, \nabla_{3}\right)\right)=0 .
$$

By the Jacobi identity and (4.3), 


$$
b\left(\Phi,\left(\nabla_{0}, \nabla_{1}\right)\right)+a\left(\Phi,\left(\nabla_{2}, \nabla_{3}\right)\right)=0 .
$$

By (2.11),

$$
b\left(\Phi, G_{01}\right)+a\left(\Phi, G_{23}\right)=0 .
$$

Finally by (A6) and (A7) one sees that (A8) is satisfied identically, as it should.

By a similar reduction of (A5) one finds

$$
a^{2}+b^{2}=1 \text {. }
$$

The preceding argument holds any for any algebra including the loop algebra.

'G. 't Hooft, Nucl. Phys. B 79, 276 (1974).

${ }^{2}$ A. M. Polyakov, JETP Lett. 20, 194 (1974).

${ }^{3}$ B. Julia and A. Zee, Phys. Rev. D 11, 2227 (1975).

${ }^{4} J$. Schwinger, Science 165, 757 (1969).
${ }^{5}$ For a field theory example of noding solitons, see R. Finkelstein, R. LeLevier, and M. Ruderman, Phys. Rev. 83, 326 (1951).

${ }^{6}$ A. C. Cadavid and R. Finkelstein, Lett. Math. Phys. 16, 279 (1988).

${ }^{7}$ A. C. Cadavid and R. Finkelstein, Lett. Math. Phys. 17, 157 (1989).

${ }^{8} \mathrm{P}$. Goddard and D. Olive, Rep. Prog. Phys. 41, 1361 (1978).

${ }^{9} J$. Arafune, P. Freund, and C. Goebel, J. Math. Phys. 16, 433 (1975).

${ }^{10}$ E. B. Bogomolny, Sci. J. Nucl. Phys. 24, 449 ( 1979); L. D. Faddeev, Lett. Math. Phys. 7, 289 (1976).

"M. K. Prasad and C. M. Sommerfield, Phys. Rev. Lett. 35, 760 (1975). ${ }^{12}$ S. Coleman, S. Parke, A. Neveu, and C. M. Sommerfield, Phys. Rev. D 15, 544 (1977). Equations (4.4) and (4.5) in this reference follow the saturation of the Bogomolny bound. Here the argument is turned around. See the Appendix.

${ }^{13}$ T. T. Wu and C. N. Yang, Properties of Matter under Unusual Conditions, edited by H. Mark and S. Fernbach (Interscience, New York, 1969).

${ }^{14}$ R. Jackiw and C. Rebbi, Phys. Rev. Lett. 36, 1116 (1976).

${ }^{15}$ P. Hasenfratz and G. 't Hooft, Phys. Rev. Lett. 36, 1119 (1976).

${ }^{16} A$. S. Goldhaber, Phys. Rev. Lett. 36, 1122 (1976).

${ }^{17}$ A. C. Cadavid and R. Finkelstein, Int. J. Mod. Phys. A 4, 2967 (1989).

I"A. C. Cadavid and R. Finkelstein, "Affine extension of supergravity," to be published in Int. J. Mod. Phys. A. 